\title{
Testeos de validez convergente, de validez de constructo y de vali- dez externa del algoritmo David Liberman (ADL)
}

\section{Convergent validity test, construct validity test and external vali- dity test of the David Liberman algorithm}

\author{
David Maldavsky ${ }^{1}$ \\ Universidad de Ciencias Empresariales y Sociales, UCES, Argentina
}

(Recepción: Marzo 2009 - Aceptación: Julio 2009)

\begin{abstract}
Resumen
El autor expone en primer lugar un complemento de testeos previos sobre la validez convergente, luego un testeo de validez de constructo y por fin un testeo de validez externa del algoritmo David Liberman. El primero de estos testeos se centra en un aspecto complementario, la sensibilidad diferencial del ADL 1) en una comparación externa (con otros métodos), y 2) en una comparación interna (entre dos modos de empleo del mismo método, el ADL). El testeo de validez de constructo expone los conceptos subyacentes al ADL, su operacionalización y algunas rectificaciones derivadas de los numerosos estudios empíricos realizados. El testeo de validez externa examina la posibilidad de emplear la investigación de caso único y su relación con la investigación de una muestra más amplia.

Palabras clave: Algoritmo David Liberman, validez convergente, validez de constructo, validez externa.
\end{abstract}

\begin{abstract}
The author first exposes a complement of a previous test about convergent validity, then a construct validity test and finally an external validity test of the David Liberman algorithm. The first part of the paper focused on a complementary aspect, the differential sensitivity of the DLA 1) in an external comparison (to other methods), and 2) in an internal comparison (between two ways of using the same method, the DLA). The construct validity test exposes the concepts underlined to DLA, their operationalization and some corrections emerging from several empirical studies we carried out. The external validity test examines the possibility of using the investigation of a single case and its relation with the investigation of a more extended sample.
\end{abstract}

Key words: David Liberman algorithm, convergent validity, construct validity, external validity.

1 Correspondencia a: David Maldavsky. Dirección postal: República Árabe Siria 3319, 5³, Buenos Aires, Argentina. C.P: 1425 Telefono: 5411-4802- 3842 E-Mail: dmaldavsky@elsitio.net 


\section{Introducción}

En este trabajo pretendemos exponer los resultados de nuestros testeos de validez convergente de la sensibilidad clínica, de validez de constructo y de validez externa del algoritmo David Liberman (ADL). Estos trabajos hacen de complemento de otros dos, uno dedicado al testeo global de validez convergente (Maldavsky, 2008d) y el otro al testeo de validez pre y postdictiva del mismo método, el algoritmo David Liberman (Maldavsky, 2008e). En realidad, el primero de los apartados del presente trabajo es en buena medida una continuación del estudio global de validez convergente previo, con nuevas especificaciones, referidas a la sensibilidad diferencial de dos estrategias (una cualitativa y la otra estadística) en el empleo del método, para detectar la fineza de los matices en los estudios clínicos.

\section{Testeo de validez convergente: la sensibilidad del algoritmo David Liberman (ADL)}

Recientemente hemos realizado dos pruebas de investigación convergente para estudiar 1) el grado de fineza con que el algoritmo David Liberman detecta los rasgos de los pacientes y 2) el modo de organizar el conjunto de los hallazgos en una presentación coherente.

En uno de los trabajos realizamos (Maldavsky, 2008b) un extenso testeo de validez convergente consistente en el contraste entre las investigaciones desarrolladas por siete equipos de investigación sobre una primera entrevista y nuestro propio estudio. Desarrollamos dos abordajes en el contraste: por un lado, comparamos nuestras conclusiones con las de cada uno de los otros estudios, y por otro lado las comparamos con todas las otras reunidas (comparación Rashomon-like). La comparación del primer tipo (trabajo a trabajo) nos llevó a advertir que entre los siete estudios previos dedicados al caso había conflictos y divergencias. Mientras que un grupo de estudios, sobre todo el de Rosenberg et al (1994), destacaban los aspectos más regresivos del caso, sin prestar atención a sus aspectos más funcionales, otro grupo de trabajos, sobre todo el de Perry (1994), privilegiaron los aspectos más bien neuróticos y funcionales de la paciente, combinados con un rasgo más bien ligado con la dependencia afectiva. El conflicto que hemos descrito tiene fuertes afinidades con un conflicto similar que se hizo evidente en el análisis con el ADL, entre dos modos alternativos de destacar lo dominante: o bien el núcleo "pasional” o bien otro sustrato, más regresivo. En dicho conflicto, en la investigación de 2008 sobre la entrevista destacamos finalmente la prevalencia de este último componente, en sintonía con la posición de Rosenberg et al., y no con la de Perry. Esta sintonía con la posición de Rosenberg et al. se complementaba con una sintonía con Perry en relación con la más matizada presentación de los componentes (deseos y defensas) detectados en el análisis de la entrevista, sin prestar atención a qué es lo prevalente. La comparación del segundo tipo (entre nuestros resultados y los de los 7 trabajos reunidos) llevó a concluir que las diferencias globales se hallan en que el ADL ha considerado 1) un ordenamiento más claro de las jerarquías internas entre los componentes, 2) el estado de las defensas según se trate de las relaciones extratranferenciales o del vínculo paciente-terapeuta.

Es conveniente aclarar estas conclusiones del testeo de validez convergente. El ordenamiento de las jerarquías internas entre los componentes de un funcionamiento psíquico permite decidir cuál será la orientación clínica del terapeuta, e incluso anticipar algunas consecuencias de ello. Los estados de la defensa tienen fuerte importancia para decidir sobre la severidad mayor o menor de un caso, ya que cuando una defensa patógena es exitosa existen mayores riesgos clínicos y mayores resistencias al tratamiento que cuando estas fracasan. Suele ocurrir, además, que, si bien el paciente relata episodios de su vida cotidiana que indiquen el fracaso de dichas defensas, durante las sesiones dichos mecanismos se vuelven exitosos. Este es precisamente el aspecto que los otros métodos no pudieron captar.

Un segundo testeo de validez convergente tuvo como objetivo ya no comparar los resultados ajenos y propios sino dos estrategias de aplicación del algoritmo David Liberman, una cualitativa y otra cuantitativa. El objetivo de este testeo era averiguar cuál de los dos resultados (el cualitativo o el estadístico) captaba con mayor riqueza los matices del funcionamiento psíquico de la paciente. Entonces en el estudio de la muestra recién mencionada (que habíamos estudiado aplicando el 
algoritmo David Liberman con un enfoque cualitativo) recurrimos a un nuevo instrumento, de carácter estadístico. Para ello nos fue necesario primero exponer y justificar (Maldavsky, 2008b) un criterio para puntuar los defensas, su estado, etc., detectados en el estudio precedente. Una vez aplicado este criterio al estudio cualitativo precedente, comparamos (Maldavsky, 2008c) los resultados correspondientes tanto en el nivel del relato como en el de los actos del habla. En el nivel del relato se advierte que existen coincidencias solo parciales entre los resultados de ambos análisis en cuanto a las pulsiones y las defensas tanto patógenas cuanto funcionales. En efecto, el análisis estadístico arroja porcentajes que otorgan cierta importancia a ciertos rasgos caracterológicos (evitativo-ambiciosos) que no fueron suficientemente valorados en la aplicación de la estrategia cualitativa. Además, estos resultados estadísticos ponen en evidencia una mayor variedad de recursos defensivos funcionales de la paciente que los detectados en el estudio cualitativo. Por fin, otra diferencia corresponde al estado de una defensa (desmentida), que en nuestra investigación inicial consideramos sobre todo como fracasado, mientras que en el análisis estadístico resultó exitoso. Esta última diferencia deriva de que un modo diferente de privilegiar una escena central del relato de la paciente: en el estudio estadístico tomamos en cuenta solo la defensa dominante, mientras que en el estudio cualitativo consideramos mayor variedad de rasgos. Quizá sea necesario agregar a nuestra propuesta de puntuación la consigna de que, en el estudio de la escena o escenas centrales, es conveniente incluir los puntajes de otras pulsiones y defensas, y no solo los que corresponden a lo principal. En cambio, la comparación entre los resultados de ambos estudios en el nivel de las escenas desplegadas arrojó coincidencias.

Es conveniente valorar las conclusiones de esta segunda investigación convergente, en la cual confrontamos los resultados de nuestra investigación previa con el ADL y los resultados obtenidos con los recursos estadísticos. Suele afirmarse que una conclusión satisfactoria de este tipo de investigación convergente consiste en que se advierten ciertas coincidencias básicas entre los resultados de ambas investigaciones, pero también algunas diferencias, que justifican la existencia de los instrumentos que se pretende testear, en este caso los recursos estadísticos. Precisamente, hemos puesto de manifiesto algunas ventajas obtenidas con el análisis estadístico, sobre todo en cuanto a evidenciar de manera más precisa y fina la coexistencia de diferentes matices en el funcionamiento psíquico de la paciente, los cuales a su vez pueden ser organizados en un conjunto coherente.

Concluimos que la estrategia estadística de aplicación del algoritmo David Liberman a un material clínico arroja resultados más matizados que la estrategia cualitativa. Esta conclusión es contra-intuitiva, y abre las puertas a la posibilidad de desarrollar estudios clínicos sistemáticos de tipo estadístico que al mismo tiempo acuerden con los fundamentos teóricos freudianos.

\section{Testeo de validez de constructo del algoritmo David Liberman (ADL)}

En nuestro testeo de validez de constructo del ADL 1) discutiremos la pertinencia teórica de la selección de los conceptos que están en la base de los instrumentos y la creación de los correspondientes inventarios, 2) describiremos la forma de operacionalizar los conceptos y 3) examinaremos si los estudios empíricos realizados confirman o conducen a rectificar parcial o totalmente la pertinencia de dicha selección.

Conceptos e inventarios. Comencemos con el problema de la pertinencia teórica de la selección de los conceptos que están en la base del ADL y la forma de construir el inventario correspondiente. Los conceptos a los que aludimos corresponden a los componentes nucleares del pensamiento freudiano con los cuales se intenta dar cuenta de las manifestaciones clínicas. Algunos autores (Luborsky et al., 1990; Perry, 1989; Holt, 2007; Perron, 2006; Maldavsky, 2003; entre otros) proponen que estos conceptos son los deseos y (excepto Luborsky et. al., 1990) las defensas. Estos son pues los conceptos básicos que el algoritmo David Liberman pretende estudiar. La investigación sobre deseos y defensas durante las sesiones requiere de un sistema categorial que permita clasificar el discurso y otras manifestaciones. Este sistema puede ser construido utilizando tanto un modo inductivo como deductivo. El camino inductivo tiene el problema de abandonar el sustento teórico psicoanalítico. El camino deductivo apela a la teoría como fundamento para realizar la selección y la definición de los conceptos principales. 
Es conveniente distinguir entre la pertinencia de uno y otro concepto, deseo y defensa y sus correspondientes inventarios. Comenzaremos con la discusión de la pertinencia del concepto deseo y del modo de crear el correspondiente inventario en el ADL. El CCRT (Luborsky, et al., 1990) fue construido utilizando un modelo inductivo, y el ICF (Perry, 1990) se apoya en una orientación deductiva, basada en las ideas de Erikson (1950). Como el constructo del ICF, el del ADL fue creado por un camino deductivo, pero a partir de la teoría freudiana. Esta orientación coincide con las propuestas de Holt y Perron. Holt (1989) en los Estados Unidos y Perron (2006) en Francia recientemente sugirieron que el sistema conceptual para la categoría de los deseos debería estar basado en la teoría freudiana de las pulsiones. Holt describió los componentes del sistema, es decir las pulsiones libidinales y agresivas, pero no operacionalizó el concepto ni construyó instrumentos a partir de esta base, y Perron solo aplicó el marco conceptual freudiano a una muestra (las respuestas a un test proyectivo). El inventario de los deseos que propone el ADL se apoyó en la categorización de Freud (1905d, 1933a) de las pulsiones eficaces en las manifestaciones clínicas. Esta decisión implica también justificar por qué no se incluyen en este inventario de deseos a los correspondientes, entre otras, a la pulsión escópica y a la pulsión invocante. La justificación para ello es que estas pulsiones no han sido consideradas por Freud como participantes en una fijación eficaz para la producción de manifestaciones clínicas específicas. En consecuencia, es posible presentar al ADL en el marco de las discusiones actuales sobre los instrumentos de investigación sistemática, mostrando similitudes y puntos de deslinde con otros métodos. En efecto, el constructo del concepto deseo que está en la base de los instrumentos del ADL fue creado deductivamente, a diferencia de lo que ocurre con el CCRT, ha partido desde las premisas freudianas, a diferencia del ICF, y ha avanzado hacia una operacionalización, a diferencia de las propuestas de Holt y de Perron.

Podemos encarar ahora el examen del otro concepto, el de defensa, y del modo de crear el inventario. La definición de defensa así como su inventario suelen partir de la clásica teoría de Anna Freud (1980), a la cual diferentes autores (Vailiant, 1992; Perry, 1989; Mc Dougall, 1989; Lacan, 1958) propusieron agregar nuevos ítems, clasificaciones, etc. De allí surgieron varios instrumentos para la investigación de las defensas, con la correspondiente operacionalización de los conceptos (Perry, 1989 y Cramer, 2006). Sin embargo en dichos inventarios parecen presentarse problemas conceptuales ligados con la teoría, con la psicopatología y con la clínica. Consideremos primero el problema ligado con la teoría. Dichos inventarios agrupan las defensas en conjuntos que corresponden a modos más o menos integrados de funcionamiento psíquico, pero no distinguen en cada conjunto cuáles son las defensas centrales y cuáles las secundarias. Por ejemplo, para Freud la proyección puede ser una defensa secundaria a la represión (en la creación de una fobia), a la desmentida (en pacientes que toman a otro como su doble), y a la desestimación (en los procesos alucinatorios aterradores).

Desde el punto de vista psicopatológico, dichos inventarios no conceden un lugar diferencial, específico, a las defensas que aparecen por un lado en las psicosis y por otro lado en las afecciones psicosomáticas, las adicciones, etc. Con respecto a las situaciones clínicas, Freud concedió mucha importancia no solo al tipo de defensa sino también a su estado, que puede ser exitoso, fracasado, etc. El síntoma histérico, por ejemplo, surge ante el fracaso de la represión, y la alucinación aterradora surge ante el fracaso de la desestimación de la realidad y la instancia paterna y el correspondiente retorno de lo desestimado. Un último punto, de gran importancia, requiere una consideración adicional. Los métodos que se basan en los conceptos de deseo y defensa no presentan articulaciones sistemáticas entre ambos, a diferencia del ADL. En efecto, este último método establece una combinación entre los dos conceptos centrales (deseo y defensa) aprovechando de la propuesta freudiana de que las defensas son destinos de la pulsión en el yo. En consecuencia, podemos decir que, como los otros métodos de investigación de la defensa, el inventario del ADL fue creado deductivamente, a partir de conceptos teóricos, psicopatológicos y clínicos, lo cual condujo a distinguir entre defensas centrales y secundarias, entre las defensas propias de determinadas patologías (como las psicosis y las afecciones psicosomáticas) y entre estados (exitoso, fracasado, etc.) de la misma defensa, y además permitió establecer un enlace fuerte de este concepto con el de deseo. 
Operacionalización de los conceptos. El paso siguiente es el estudio de las vías de operacionalización de uno y otro concepto. Comencemos con el concepto “deseo”. Suele aceptarse que es posible estudiar el deseo en escenas o episodios significativos. Para operacionalizar el deseo y detectarlo en las escenas el ADL se ha basado en la conceptualización freudiana de las vivencias de satisfacción y de dolor. En efecto, Freud sostuvo que estas vivencias son las que permiten enlazar a las pulsiones con el mundo de las representaciones y los pensamientos. Toda vivencia tiene tres componentes: 1) percepción, 2) motricidad, 3) afecto. En consecuencia, el ADL propuso que para cada pulsión cada uno de estos tres componentes tiene rasgos diferenciales, como por ejemplo la percepción de ritmos o golpes para la libido intrasomática, la percepción puntiforme para el erotismo oral primario, la captación de expresiones faciales o equivalentes para el erotismo sádico oral secundario, etc. Este paso intermedio permitió el pasaje desde el concepto "pulsión" al de los deseos específicos, los cuales pueden ser detectados en las escenas. El argumento que justifica el enlace entre 1) los deseos inventariados previamente y 2) las escenas es precisamente el concepto de vivencias, de satisfacción o de dolor. Con ello se abre la posibilidad de crear grillas que especifican los rasgos propios de los episodios o las escenas como expresión de uno u otro deseo.

Este primer paso en la operacionalización, que permite crear instrumentos para la detección de los deseos en las escenas, se acompaña de un segundo paso, que corresponde a la operacionalización del concepto "defensa”. En los hechos, la operacionalización del concepto “defensa” se presenta como una serie de pasos sucesivos para responder a diferentes preguntas con lo cual el investigador se orienta en la toma de decisiones científicas. Algunas preguntas tienen que ver con la defensa central y otras con los mecanismos complementarios. Consideremos primero las preguntas referidas a la defensa central. La primera de ellas es si la defensa se opone al deseo o a la realidad y la instancia paterna. La respuesta a esta pregunta conduce a una bifurcación de los caminos, ya que una cosa es estudiar los rasgos de la defensa ante el deseo y otra analizar las características de la defensa contra la realidad y la instancia paterna. En consecuencia es conveniente seguir cada uno de estos caminos por separado. Si la defensa se opone a la realidad y la instancia paterna, entonces cabe preguntarse si el mecanismo consiste o bien en la desmentida o la desestimación o bien en la sublimación, la creatividad o la defensa acorde a fines. Un paso ulterior en la investigación consiste en detectar si la desmentida o la desestimación es funcional o patógena. El paso siguiente consiste en decidir si la defensa patógena es la desmentida o la desestimación, y, por fin, un último paso lleva a decidir sobre el estado (exitoso, fracasado, mixto) de dicha defensa. Un ordenamiento similar de interrogantes se presenta en relación con las defensas frente al deseo: en primer lugar, decidir si el mecanismo es o bien la represión o bien la sublimación, la creatividad o la defensa acorde a fines, en segundo lugar, decidir si la represión es funcional o patógena y por último decidir sobre el estado (exitoso, fracaso, mixto) de la represión patógena. A ello se agregan las preguntas referidas a las defensas secundarias, sobre todo en cuanto a sus rasgos específicos y en cuanto a su estado (exitoso, fracasado, etc.). La respuesta a cada uno de estos interrogantes va acompañada de una argumentación, que no podemos exponer ahora. Valga como ejemplo que, para decidir si la defensa se opone a la realidad y la instancia paterna o al deseo, nos guiamos por la propuesta freudiana ya mencionada, de que las defensas son destinos o vicisitudes de las pulsiones. Por lo tanto, cada pulsión tiene destinos específicos, y la detección de los deseos correspondientes permite inferir a qué se opone la defensa central. Argumentaciones similares permiten avanzar en las respuestas a los interrogantes siguientes.

La construcción de los instrumentos específicos se apoya todavía en otro criterio obtenido deductivamente. En efecto, ya dijimos que el ADL pretende estudiar los deseos y las defensas en las escenas. Pero Freud diferenció entre dos tipos de escenas. Unas son las que el paciente relata al terapeuta y las otras son las que se despliegan en la sesión misma, que no necesariamente coinciden con las primeras. En consecuencia, nos vimos llevados a crear instrumentos para detectar los deseos en uno y otro tipo de escena. Para estudiar las escenas que el paciente narra construimos una grilla de los relatos, y para investigar las escenas que el paciente despliega en la sesión construimos tres grillas, una sobre actos del habla, otra sobre los componentes paraverbales y otra sobre los desempeños motrices. Otros instrumentos de investigación de los deseos no diferencian claramente 
entre ambos tipos de escenas (relatadas y desplegadas al hablar), y solo conocemos un método de investigación sistemática (el de Stiles ) que investiga específicamente los actos del habla, aunque con una perspectiva no psicoanalítica. A estos instrumentos que permiten detectar los deseos en las escenas se agregan otros que permiten detectar las defensas y su estado. Estos otros instrumentos consisten en instructivos que permiten decidir paso por paso entre las diferentes preguntas sobre los mecanismos mencionadas poco antes.

Hasta aquí nos referimos al estudio de las manifestaciones de los pacientes, pero a ello es conveniente agregar un instrumento para investigar el deseo y la defensa en las intervenciones del terapeuta. Para ello el ADL cuenta con un instrumento complementario, que requiere una breve explicación. Es frecuente que el terapeuta no realice relatos en sesión, por lo cual la investigación suele centrarse en las escenas que este despliega, la cual por supuesto se combina con las escenas desplegadas por el paciente. El problema es el siguiente: como el estudio de cada escena suele arrojar resultados multivariados, es necesario decidir qué es lo dominante. Para decidir al respecto en cuanto al discurso del paciente, alcanzan con algunas instrucciones referidas al análisis de las escenas relatadas o desplegadas. Pero para decidir acerca de lo dominante en el terapeuta, fue necesario categorizar sus intervenciones con una perspectiva funcional: intervenciones introductorias, centrales y complementarias. No es lo mismo que determinada combinación entre deseo y defensa del terapeuta se exprese en una intervención introductoria que en una complementaria, etc. Este otro instrumento no ha sido creado deductivamente sino de manera inductiva.

Ratificaciones y rectificaciones. Nos queda por considerar el último punto, es decir, hasta dónde la práctica de la investigación con el ADL ha llevado a introducir cambios en los conceptos o en los instrumentos. A lo largo de los últimos 10 años el ADL ha sido empleado en unas 100 investigaciones concretas, presentadas en ámbitos científicos internacionales, en revistas con referato o en el terreno académico. En términos generales, los instrumentos han puesto en evidencia su utilidad para investigar con fineza numerosos rasgos de la organización y el funcionamiento psíquico global o puntual de pacientes en tratamiento y de sujetos en contextos no clínicos. La mayor parte de los instrumentos y los conceptos siguen siendo los mismos que en un comienzo, aunque se han introducido algunos cambios puntuales que favorecen la sutileza de la investigación, mientras que otras modificaciones están en curso. En lo que sigue expondremos los principales cambios, sea en cuanto a los conceptos y los métodos. Los cambios corresponden sobre todo al inventario de las defensas centrales.

Una defensa prácticamente no ha sido detectada: la sublimación. Consideramos sin embargo que no es un error incluirla entre las defensas centrales, sino que más bien no hemos seleccionado materiales que permitan detectarla, ya que en su mayoría los estudios con el ADL son o bien clínicos o bien de problemas psicosociales. Solo uno de los trabajos compara (Maldavsky et al., 2005) las obras literarias con la producción discursiva en sesión de un paciente, y en ese caso el concepto de sublimación se reveló útil. Otra novedad fue la inclusión de una defensa central a la que denominamos acorde a fines (Maldavsky et al., 2007). Esta modificación se nos impuso cuando nos vimos llevados a investigar en el terreno psicosocial, sobre todo la actividad laboral, que no implica ni creatividad ni sublimación, pero que tampoco implica defensas patógenas. Una nueva modificación consistió en un más refinado análisis de las defensas secundarias a la desestimación del afecto, para lo cual nos resultaron de mucha utilidad los aportes recientes de los neurobiólogos (Maldavsky, 2008d) que estudian PTSD. Sobre todo nos resultó esclarecedora la descripción del mecanismo de fuga, la cual puede confundirse con la técnica evitativa de los pacientes fóbicos. Esta defensa evitativa puede aparecer o bien combinada con la represión, hecho que es frecuente en las histerias de angustia, o bien combinada con defensas acorde a fines, como se hace evidente en muchas manifestaciones de la vida cotidiana. En cambio, en otras ocasiones, como las que se presentan en las PTSD, la fuga es ciega y sin término, y además carece de valor simbólico. En tal caso corresponde más bien al mecanismo de fuga que Freud (1915c) atribuyó al yo realidad inicial, y muy a menudo tal mecanismo es complementario de la desestimación del afecto (Maldavsky et al., 2002). El fracaso en esta defensa complementaria suele conducir hacia las manifestaciones clínicas de las afecciones psicosomáticas, las adicciones, la violencia desatada, las prácticas suicidas, etc, Además, a veces 
esta defensa de fuga tiene también un carácter funcional, como puede ocurrir en ciertas situaciones críticas en que corren riesgo las bases orgánicas de los procesos psíquicos.

En cuanto a las modificaciones en los instrumentos, advertimos que tiene cierta debilidad el camino para describir e inferir los componentes paraverbales, por lo cual estamos desarrollando una categorización complementaria de la grilla correspondiente. Esta categorización pretende describir los rasgos de cada manifestación paraverbal a partir de la motricidad que genera dicha manifestación. Este criterio de descripción se apoya en la propuesta realizada por autores americanos, alemanes y austríacos para estudiar las expresiones faciales a partir de las motricidades implicadas. Respecto de la producción de los componentes paraverbales, diferenciamos tres motricidades: la que genera la columna de aire, la de las cuerdas vocales y la bucal, con funciones específicas y múltiples combinaciones posibles entre los componentes.

Esta categorización está en curso (Maldavsky \& Rembado, 2008b). Además, al testear con un juez independiente la manualización del instrumento para detectar las defensas secundarias en pacientes con rasgos patológicos de carácter advertimos algunas ambigüedades que pueden resultar desorientadoras a la hora de intentar decidir acerca del rasgo funcional o patógeno de dichas defensas. Recientemente hemos presentado propuestas (Maldavsky \& Wainer 2008c) para subsanar este problema. Otra modificación corresponde al diccionario computarizado para el análisis de las palabras. Con todo, no se trata de una rectificación, sino de una propuesta de enriquecimiento, por la cual, a las 600.000 palabras ya existentes, se le agregarán unas 50.000 adicionales. El proyecto está también en curso.

\section{Testeo de validez externa de la investigación clínica con el algoritmo David Liberman (ADL)}

Otro tipo de validez que deseamos encarar, más brevemente, es el referido al grado de extensión que pueden tener los resultados de nuestros estudios clínicos. Es decir, nos referimos al problema de la validez externa de los resultados obtenidos con el ADL al estudiar una sesión, un período de un tratamiento o la terapia en su totalidad. En efecto, se suele sostener que la investigación de caso único no arroja resultados que pueden ser extensibles a otros casos o a una población más amplia. A ello se puede responder que el método de la investigación clínica es como el de la cartografía: un explorador releva un territorio y realiza un primer mapa, que otro rectifica y enriquece, y un tercero precisa en algunos detalles, etc. Algo similar suele ocurrir cuando diferentes autores estudian el mismo tipo de problema clínico, y cada uno rectifica y enriquece los aportes de los precedentes, siempre y cuando no interfieran las terminologías idiosincrásicas y las arrogancias recíprocas (Widlöcher, 2001).

En esta oportunidad deseamos aportar de otro modo a la discusión respecto de la representatividad de una investigación de caso único, por el contraste entre sus resultados y los obtenidos en una investigación con un diseño diferente, basada en una muestra de 10 casos.

En un trabajo presentado en un Meeting de la SPR (Maldavsky et al., 2006) investigamos los deseos y las defensas (así como su estado) predominantes en 10 sujetos soriásicos. Aplicamos el ADL a las respuestas que estos daban al Test de Phillipson. Los entrevistados nunca habían estado en psicoterapia. La enfermedad tenía diferentes grados de gravedad: 1) episódica, 2) crónica, bajo control, 3) crónica y no controlable. Los resultados de los estudios de los deseos y las defensas que detectamos tenían algunas similitudes y diferencias. El núcleo era el mismo, compuesto por LI y la desestimación del afecto y O1 y la desmentida. Otros deseos y defensas eran complementarias: 1) FU y la represión + evitación, 2) A1 y la desmentida, 3) O2 y la desmentida. Cuando el sistema defensivo tenía éxito, la enfermedad era menos grave que cuando estos mecanismos fallaban. Además, cuanto mayor la gravedad del caso, más pobre era el repertorio de los deseos expresados en el discurso.

Con posterioridad investigamos con detenimiento una sesión de Diego, un paciente soriásico cuyos síntomas en ese momento se habían atenuado notoriamente (Maldavsky et al., 2008a). La muestra de dicha investigación difiere del estudio de los 10 casos. El nuevo estudio se centro en 
una sesión. Además, el paciente concentró su atención en sus problemas y conflictos, mientras que quienes respondieron al test solo en unas pocas ocasiones aludieron a historias de su propia vida. En tercer lugar los sujetos incluidos en la muestra a la que se administró el test tenían un diferente grado de gravedad en su enfermedad, mientras que el paciente en sesión aludió en sus relatos a varios estados, más o menos graves, de sus perturbaciones somáticas.

Estos tres hechos (el contexto en que se obtuvieron las muestras, la mayor o menor referencia a cuestiones personales en los relatos, la diferencia entre los grados de gravedad de la afección somática) conduce a anticipar que los resultados de las dos investigaciones no coincidirían.

En ambas investigaciones los resultados obtenidos consistieron no en una formulación única sino en un espectro complejo en el cual se combinan varios deseos y defensas (y sus estados), que eran específicos para cada caso o para cada momento de la sesión. Al comparar ambos estudios se advierte que los deseos y las defensas centrales eran los mismos. Las diferencias entre los resultados de ambos estudios consisten sobre todo en el estado de la defensa (Maldavsky et al., 2008a).

En la primera investigación (sobre los 10 casos), entre los deseos y las defensas detectamos, según ya lo indicamos, LI y la desestimación del afecto, O1 y la desmentida, O2 y la desmentida, A1 y la desmentida, FU y la represión + rasgos evitativos de carácter. En la segunda investigación (de caso único) detectamos los mismos deseos y defensas con la adición de A2 y la defensa acorde a fines y FG combinada a veces con la represión + rasgos histriónicos de carácter y a veces (producción onírica) con la creatividad.

En las dos investigaciones los dos deseos y las defensas dominantes fueron LI y la desestimación del afecto y $\mathrm{O} 1$ y la desmentida. Cuando estos se combinaban con FU y la represión exitosa + rasgos evitativos de carácter, también la desestimación del afecto y la desmentida tenían éxito. Pero cuando los rasgos evitativos de carácter eran exitoso/ fracasados, lo mismo ocurría con las defensas centrales y cuando los primeros fracasaban, también pasaba esto con los mecanismos centrales.

Los resultados del análisis de algunas anécdotas del paciente y de algunas escenas desplegadas en las que predominaban LI y la desestimación exitoso/fracasada del afecto armonizaban con los resultados de los estudios del grupo más grave de la muestra de los 10 casos.

El resultado del análisis de otros relatos del paciente coincidía con el resultado del estudio del grupo intermedio de la muestra. Por fin, el resultado del estudio de las anécdotas de los integrantes de la muestra con una situación clínica más benigna conducían a distinguir entre el pasado (con un incremento de la soriasis) y el presente (con una disminución de la enfermedad). El resultado del análisis del primer momento (pasado) coincidía con el resultado del análisis del estudio de las situaciones conflictivas del paciente con su esposa. El resultado de la investigación del momento presente, en cambio, coincidía con el resultado del estudio de la situación actual de Diego con su esposa.

En consecuencia, es posible inferir que los resultados de estas dos investigaciones coinciden. La comparación que acabamos de realizar pone en evidencia los problemas que se presentan cuando se pretende compatibilizar (como es nuestra intención) un estudio detenido de un caso con un enfoque que reúne varios de ellos. El estudio de este caso ofrece resultados multivariados para cada momento de una sesión, y como además se producen cambios clínicos (positivos o negativos), dichos resultados también suelen variar. Por lo tanto, parece conveniente comparar los casos clínicos de un modo fragmentario: un momento de un paciente puede coincidir con otro de un segundo paciente, o de una muestra más amplia, etc. Freud tendía además a destacar la singularidad de cada caso, sus diferencias con respecto a los demás, las cuales a su vez pueden asemejarse a otros casos (Maldavsky, 2008e y 2008f). Aun así, como los casos están reunidos por un rasgo (como la soriasis), es posible intentar hallar los componentes similares a todos ellos, tal como lo expusimos en nuestro trabajo. Además, los resultados de esta comparación entre dos investigaciones conduce a sugerir que en los estudios diagnósticos es conveniente describir los resultados en términos de diferentes formas de organización psíquica del paciente entre las cuales este puede alternar (por ejemplo, una organización psíquica en la cual predomina una corriente psíquica psicótica, que puede alternar 
con otra organización psíquica en la cual prevalece una corriente psíquica más acorde a fines, etc. (Maldavsky et al., 2008g). Esta propuesta requiere de una metodología compleja de investigación, afín con el enfoque clínico psicoanalítico y con los requisitos de la investigación sistemática.

\section{Referencias}

Cramer, P. (2006). Protecting the self; defense mechanisms in action. Nueva Cork: Guilford.

Erikson E. (1950). Infancia y Sociedad. Buenos Aires: Editorial Paidos.

Freud, A. (1980). El Yo y los mecanismos de defensa. Barcelona: Editorial Paidós.

Freud, S. (1905d). “Tres ensayos de teoría sexual”. AE, vol. 7.

Freud, S. (1915c). “Pulsiones y destinos de pulsión”. AE, vol. 14.

Freud, S. (1933a). “Nuevas conferencias de introducción al psicoanálisis”. AE, vol. 22.

Holt, R. R., (2007). Primary Process Scoring Manual. Handbook of Clinical Scoring Systems for Thematic Apperceptive Techniques Taylor and Francis, NY.

Lacan, J. (1958). “La dirección de la cura y los principios de su poder”. Escritos, vol. I.

Luborsky, L., y Crits-Christop, P. (1990). Understanding Transference: The CCRT Method. New York: Basic Books.

Mc Dougall (1989). Teatros del cuerpo. Madrid: Yebenes.

Maldavsky, D. (2003). La investigación psicoanalítica del lenguaje: algoritmo David Liberman. Buenos Aires: Editorial Lugar.

Maldavsky, D. (2005). Un diccionario computarizado para la investigación del discurso desde la perspectiva psicoanalítica. Revista del Instituto de Investigaciones, Vol. 10, №1.

Maldavsky, D. (2008a). Un análisis de tres niveles (estructura psíquica, intrasesión, intersubjetividad) de una primera sesión de 15 años atrás (Ms. Smithfield) usando el algoritmo David Liberman (ADL). Revista del Instituto de Investigaciones, UBA, pp. 79-116

Maldavsky, D. (2008b). Un estudio de validez convergente de los resultados de la investigación con el ADL y de su reelaboración estadística. Depsicoterapia, en prensa.

Maldavsky, D. (2008c). El enfoque clínico freudiano, la investigación sistemática con el algoritmo David Liberman (ADL) y el empleo de la estadística: un ensayo preliminar. Depsicoterapia, en prensa.

Maldavsky, D. (2008d). Testeos de validez convergente del algoritmo David Liberman (ADL), sent.

Maldavsky, D. (2008e). Testeos de validez pre y posdictiva del algoritmo David Liberman (ADL). Revista Acta Psiquiátrica, en prensa.

Maldavsky, D. et al. (1992). Teoría y clínica de los procesos tóxicos. Buenos Aires: Amorrortu Editores.

Maldavsky, D., Tate de Stanley, C., Alvarez, L., Britti, A. M., Neves, N. y Roitman, C. R. (2006.). Libidinal fixations and defenses in psoriasis illness: analysis of the discourse with DLA, SPR Meeting, Edimburgo.

Maldavsky, D., et al. (2007a). La intersubjetividad en la clínica psicoanalítica. Investigación sistemática con el algoritmo David Liberman (ADL). Buenos Aires: Editorial Lugar. 
Maldavsky, D., Alvarez, L., Manson, M. (2008a). Sobre las fijaciones pulsionales, las defensas y su estado en un paciente con psoriasis, estudiado con el Algoritmo David Liberman (ADL). Revista Subjetividad y Procesos Cognitivos, vol. 11, pp. 114-135.

Maldavsky, D., Rembado, J. M. (2008b). Estado actual del diseño en curso de un instrumento en el marco del método psicoanalítico ADL. IV Jornadas de Investigación en Psicología y VI Jornadas de Actualización del algoritmo David Liberman, UCES, 2008.

Maldavsky, D. y Wainer, A. (2008c). Revisión de un procedimiento del ADL para la detección de un tipo de defensas en el nivel del relato. IV Jornadas de Investigación en Psicología y VI Jornadas de Actualización del algoritmo David Liberman, UCES, 2008.

Maldavsky, D. et al. (2008e). El caso Z (Donnet y Green) revisado. Investigación sistemática de las erogeneidades y las defensas en el contexto intersubjetivo con el algoritmo David Liberman (ADL). Clínica e Investigación relacional, Revista electrónica de Psicoterapia, vol. (1), 1, pp. 192-224.

Maldavsky, D. (2008f). Sobre las investigaciones de caso único y las combinaciones entre ellas, con especial referencia a la perspectiva de la intersubjetividad. Estudios sistemáticos con el algoritmo David Liberman (ADL). Clínica e Investigación relacional, Revista electrónica de Psicoterapia, vol. (2), 1, pp.185-219.

Maldavsky, D., Roitman C., Tate de Stanley, C., (2008g). Correntes Psíquicas e Defesas: pesquisa sistemática de conceitos psicanalíticos e da práctica clínica com o algoritmo David Liberman (ADL). Revista Psicanálise, vol. 10, nº 1, pp. 31-68.

Perrron, R. (2006). Decir, no decir, decir de otro modo. Subjetividad y procesos cognitivos, $\mathrm{n}^{\circ} 10$, UCES, Buenos Aires, en prensa.

Perry, J. C., Augusto, F. y Cooper, S. H. (1989). Assessing psychodynamic conflicts: 1. Reliability of the Idiographic Conflict Formulation Method, Psychiatry, 52, 289-301

Perry, J. C. (1990). The Defense Mechanism Rating Scale manual. (5th ed.). Cambridge, MA: Copyright by J. C. Perry, MD.

Vaillant, G. (1992). Ego mechanism of defense: a guide for clinicians and researchers. Washinton DC: American Psychiatric Press.

Widlöcher, D. (2001). Sobre la cuestión de la refutabilidad y la investigación clínica planificada en psicoanálisis. Revista Subjetividad y Procesos Cognitivos, UCES, vol. 1, pp. 219-230. 